\title{
Heartbeat: Do common genetic variants have a role in cardiovascular disease prediction?
}

Estimates, or risk scores, of the likelihood of future cardiovascular disease (CVD) in healthy people are widely used to guide primary preventative medical therapy. Current risk scores rely mainly on phenotypic criteria, such as serum lipid levels, and predictive accuracy is suboptimal, particularly in patients with intermediate risk scores. Genome-wide studies now have identified numerous single nucleotide polymorphisms (SNPs) associated with CVD but the potential additive value of these common genetic variants for CVD prediction is not clear.

In this issue of Heart, Morris and colleagues (see page 1640) calculated a risk score that combined a standard phenotypic risk estimate with analysis of 53 common SNPs. This score was then evaluated using data from several prospective studies including a total of nearly 12 thousand people initially free of CVD who experienced 1444 incident CVD events during 10 years of follow-up. Overall, the addition of SNP data did not improve CVD risk prediction signficantly. However, in those at intermediate risk based on phenotypic criteria, the authors suggest that "genetic information would prevent one additional event for every 462 people screened" (figure 1 ).

The lack of a major improvement risk stratification using genetic markers certainly is disappointing at first glance. However, we should keep in mind, as Pereira discusses in the accompanying editorial (see page 1612), that there are many reasons why this gene score was not as helpful as hoped. First, CVD is multifactorial so that although 53 SNPs seems like a lot, we may need many more for accurate risk prediction. Second, the effect size for each of these SNPs is small and may be modulated by concurrent genetic and phenotypic factors. Finally, "another possibility that we should not leave unexplored is that it may be that our clinical thinking and atavic mode of action is too much shaped by the historical way of developments of the tools we use for prediction, diagnosis and

Correspondence to Professor Catherine M Otto, Division of Cardiology, University of Washington, Seattle, WA 98195, USA; cmotto@u.washington.edu

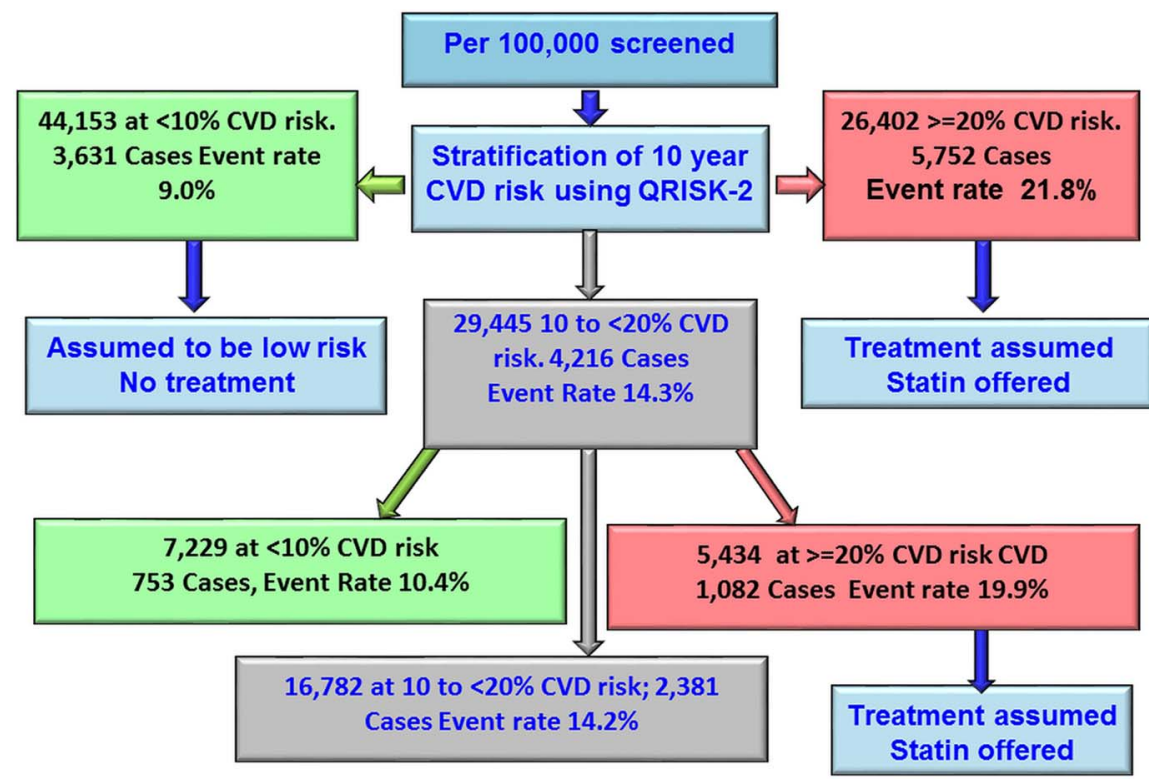

Figure 1 Flow chart showing the modelling of reclassification using Gene Score. CVD, cardiovascular disease.

treatment .... [and] we fail to think of genetic risk factors as something conveying information that is not exactly the same as state-like variables such as blood glucose or blood pressure." He concludes: "The use of polygenic information on modelling the occurrence of cardiovascular events will eventually gain much more nuance and come with more biological information, allowing for a more accurate risk prediction and more specific and timely institution of preventive measures."

There is scant evidence to guide our management of patient with cardiac disease undergoing non-cardiac surgery,

particularly for less common cardiac conditions such as hypertrophic cardiomyopathy (HCM). In an observational cohort study of 92 HCM patients undergoing noncardiac surgery at a tertiary medical center, Dhillon and colleagues (see page 1627) found that only 5\% of HCM patients suffered death, myocardial infarction or stroke within 30 days. However, a composite secondary endpoint of decompensated heart failure, re-hospitalization and atrial fibrillation was more common in HCM patients compared to controls (22\% vs $12 \%, \mathrm{p}-0.03)$ (table 1$)$. The authors conclude that non-cardiac surgery

Table 1 Thirty-day outcomes in the two subgroups

\begin{tabular}{lllc}
\hline & $\begin{array}{l}\text { Patients with HCM } \\
(\mathbf{n}=92)\end{array}$ & $\begin{array}{l}\text { Patients without HCM } \\
(\mathbf{n}=184)\end{array}$ & p Value \\
\hline $\begin{array}{l}\text { Composite events } \\
\text { Individual primary events }\end{array}$ & $20(22 \%)$ & $22(12 \%)$ & 0.03 \\
Death & $4(4 \%)$ & $3(1.6 \%)$ & 0.3 \\
Myocardial infarction & $1(1 \%)$ & $1(0.5 \%)$ & 0.5 \\
Stroke & 0 & $2(1 \%)$ & 0.6 \\
CHF during surgical admission & $4(2 \%)$ & $2(1 \%)$ & 0.1 \\
CHF rehospitalisation within 30 days postoperatively & $15(16 \%)$ & $16(9 \%)$ & 0.01 \\
\hline CHF, congestive heart failure; HCM, hypertrophic cardiomyopathy. & &
\end{tabular}

Heart October 2016 Vol 102 No 20

1605 

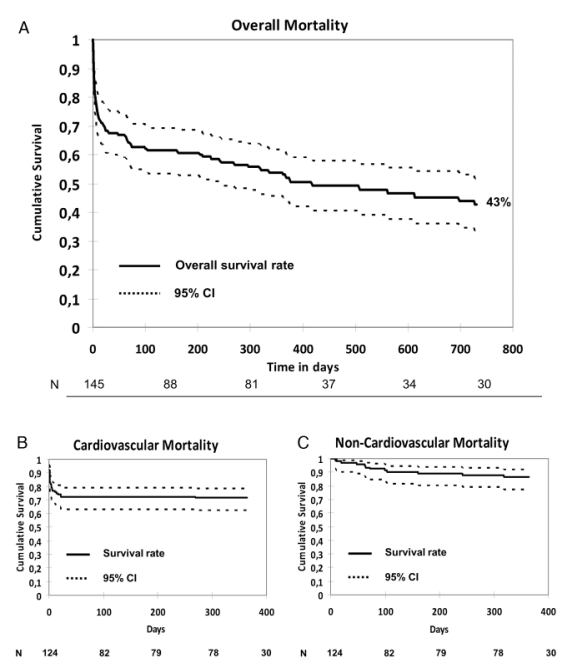

Figure 2 Kaplan-Meier curves of survival after primary percutaneous coronary intervention for overall mortality (A) and 1-year cardiovascular (B) and non-cardiovascular (C) mortality.

can be performed safely in HCM patients at experienced medical centers if hemodynamics are carefully monitored with optimization of loading conditions.

Prof. Caramelli puts this paper in perspective in an editorial (see page 1610) in which he points out that the rate of surgery is very high on worldwide basis, averaging 3.2 surgeries per person over a lifetime. Further, he suggests that perioperative care should be a new cardiology subspecialty: "To deliver proper care, the perioperative cardiology doctor must have different skills, which include knowledge about very specific issues like intensive care, acute coronary syndromes, thrombosis, cognitive function, cardiovascular consequences of renal or pulmonary dysfunction, and core clinical aspects of the disease." He suggests the peri-operative cardiovascular specialist is like a chameleon: "The chameleon has characteristic eyes that are independently mobile to look at all aspects but can aim at prey while focusing on coordination, along with the ability to change colours or assume different roles. It thus seems appropriate to nickname perioperative

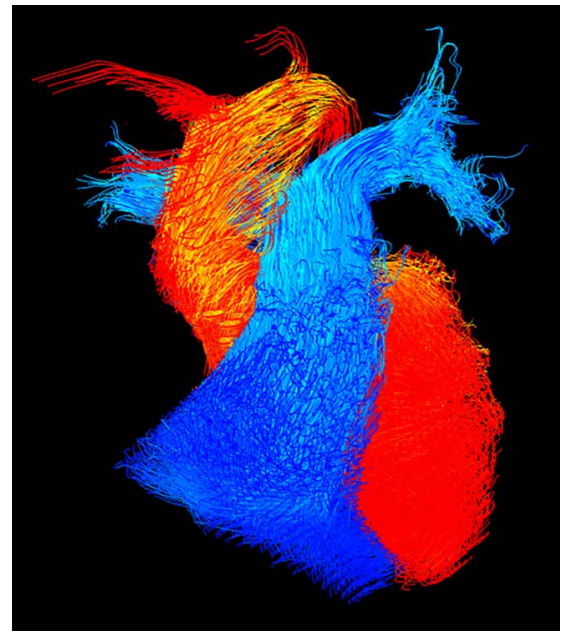

Figure 3 Judges' Choice Winner. Dr Victoria Stoll, University of Oxford Centre for Clinical Magnetic Resonance Research, University of Oxford, Oxford, UK. "The Kinetic Energies of Left Ventricular 4-Dimensional Flow Components Correlate with Established Markers of Prognosis and Represent Novel Imaging Biomarkers in Both Ischaemic and Dilated Cardiomyopathy."

cardiology physicians as chameleon doctors."

As the population ages, we see increasing numbers of very elderly patients with acute coronary syndromes (ACS). Randomized clinical trials of primary percutaneous intervention (PCI) for ST elevation myocardial infarction (STEMI) included few very elderly patients, leaving many clinicians uncertain about the balance between risk and benefit of interventional therapy in this patient subset, given a higher rate of complications and fewer remaining expected years of life. Petroni and colleagues (see page 1648) report outcomes in a multicenter retrospective study of 145 patients age 90 years or older who were treated with primary PCI for STEMI. Revascularization was achieved in $86 \%$ of cases with $60 \%$ of procedures performed via a trans-radial approach. These patients were high risk $21 \%$ were in cardiogenic shock at admission, in-hospital mortality was $24 \%$ and only $53 \%$ survived to one year (figure 2). Even so, the rate of complications was low, with significant bleeding in only $4 \%$ of patients. The authors conclude that primary PCI is appropriate in selected nonagenarians with STEMI because there is a high rate of successful reperfusion. Unfortunately, this study did not include data on all consecutive nonagenarians with STEMI so the optimal criteria for for selecting which patients should receive PCI remain unknown. In addition, outcomes can only be compared to "expected outcomes" based on previous publications. Nevertheless, the current study shows that primary PCI is reasonable for STEMI in nonagenarians, next we need a prospective randomized trial in this patient group.

The Education in Heart article in this issue (see page 1680) summarizes the current approach to diagnosis and management of pulmonary hypertension. There are several key points: (1) pulmonary hypertension is classified into 5 groups based on cause and treatment options, (2) echocardiography is a useful screening tool but right heart catheterization is needed to make a definitive diagnosis, (3) medical therapy is targeted towards one of 3 pathways (nitric oxide, endothelin and prostacyclin), (4) patients with systemic sclerosis should be screened for pulmonary hypertension, (5) chronic thromboembolic pulmonary hypertension is best treated with pulmonary endarterectomy and (6) pregnancy in patients with pulmonary hypertension is associated with high maternal mortality rates.

Be sure to take a look at the beautiful images from the British Heart Foundation Reflections on Research Competition 2016 (see page 1693) in our Cardiology in Focus section (figure 3).

The Image Challenge in this issue shows an interesting magnetic resonance scan in a 59-year-old woman with breathlessness (see page 1654).

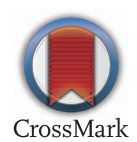

To cite Otto CM. Heart 2016;102:1605-1606.

Heart 2016;102:1605-1606.

doi:10.1136/heartjnl-2016-310515 